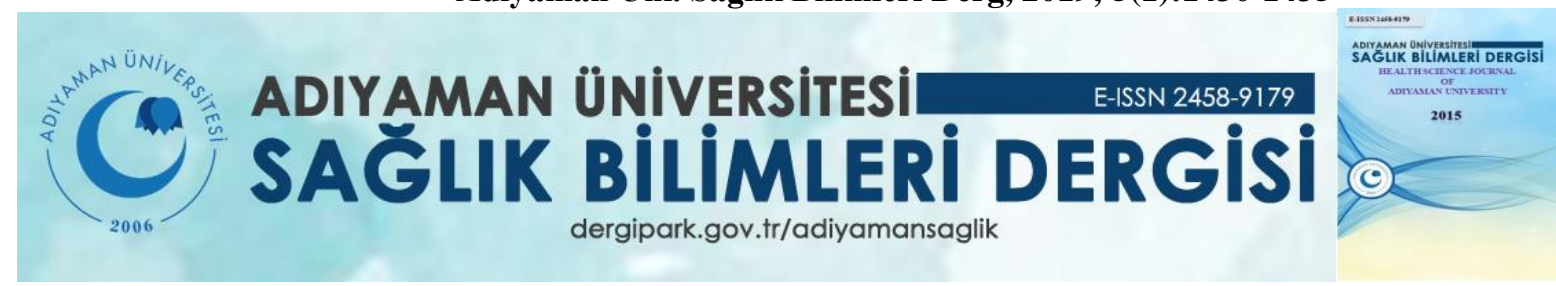

Olgu sunumu/ Case report

\title{
İliak arter girişimi için transpedal yaklaşım: Nadir bir olgu sunumu
}

\author{
Hakan KAYA $^{1}$ \\ $\ddot{\mathbf{O} z}$ \\ ${ }^{1}$ Adıyaman Üniversitesi Eğitim Araştırma Hastanesi Kardiyoloji Kliniği
}

Arteryel kan akımının yeniden sağlanması, ayak koruyucu olarak kritik ayak iskemisi olan hastaların başlıca önceliğidir. Son yıllarda, kardiyovasküler teknik ve becerilerdeki artışla ayak korumak için daha fazla operasyon yapılmıştır. Ayak arter tıkanıklığında antegrad girişimler, yaklaşık \% 20 oranında başarısızlığa neden olur. Retrograd yaklaşımlar ise genellikle diz ve diz altı arter tıkanıklıklarında uygulanır. Çok seyrek olarak femoral arter tıkıanıklıklarında da retrograd yaklaşım uygulanır. Bu vakada ise sağ external iliak arter çıkışından başlayıp popliteal artere kadar uzanan tamamen tıkanmış arter segmentine sahip bir hastada retrograd yaklaşımla başarılı bir rekanalizasyonun sağlandığı çok daha nadir bir olgu sunulmaktadır.

Anahtar sözcükler: iliak arter hastalığ, transpedal yaklaşım,

\section{Transpedal approach for iliac artery intervention: A rare case report}

\section{ABSTRACT}

Achieving arterial blood flow again is the main priority for the patients having critical foot ischemia in terms of protecting foot. In recent years, more operations have been done to save foot with the increase in cardiovascular techniques and skills. Antegrade attempts in foot artery occlusion result in failure approximately by $\% 20$. Retrograde approaches are usually applied to the popliteal and infrapopliteal arteries. Very rarely, retrograde approach is applied in femoral artery occlusions. In this case, a very rare case provided with successful recanalization through a retrograde approach for a patient having fully clogged arteries from right external iliac artery outlet to popliteal artery is presented.

Key words: iliac artery disease; transpedal approach.

\section{Yazışmadan Sorumlu Yazar}

\section{Hakan KAYA}

Adıyaman Üniversitesi Kardiyoloji AD. Adıyaman. Turkey

Tel : +90 (0416) 2161015

Email: kardiyolog02@gmail.com
Doi: 10.30569/adiyamansaglik.488010

\begin{tabular}{ll}
\hline Geliş Tarihi: & 27.11 .2018 \\
\hline Kabul Tarihi: & 15.12 .2018 \\
\hline
\end{tabular}




\section{GíRiş}

Günümüzde iliyofemoral arter tıkanıklığında yapılan aorto-femoral köprüleme ameliyatı sayısı azalmaktadır (1). Hastaların ortalama yaşının yükselmesi, eşlik eden hastalık sıklığının artması, daha düşük komplikasyon ve daha yüksek klinik başarı nedeni ile endovasküler girişim, köprüleme cerrahisine tercih edilmektedir (2,3). Endovasküler işlem esnasında antegrad yaklaşım başarısız olduğunda retrograd yaklaşım denenmelidir $(4,5)$. Retrograd rekanalizasyon genellikle diz ve diz altı arter tıkanıklığında kullanılır. Son zamanlarda femoral arter tıkanıklığında da bu yöntem seçilmektedir. Fakat, iliak arterlerde bu yaklaşım çok nadirdir. Bu vaka, sağ external iliak arterdeki bir tıkanıklıkta balon-stent işleminin retrograd yöntem kullanılarak başarıyla yapıldığı çok nadir bir olgudur.

\section{OLGU SUNUMU}

46 yaşında kronik sigara içicisi olan erkek hasta son bir aydır giderek artan, kısa mesafeli yürüyüşlerde dinlenmesini gerektiren kramp tarzında bacak ağrıları ile kalp damar cerrahi polikliniğine başvurmuş. Hastaya 1 yıl önce diz altı arter tıkanıklığından dolayı aorto-femoral köprüleme operasyonu yapılmış. Yapılan klinik değerlendirmede sağ ayak; dorsalis pedis ve arka tibial arter nabazanlarında azalma, bilgisayarlı tomografik anjiyografide sağ external iliak arterden popliteal artere kadar tam tıkanıklık, ön tibial ve tibioperoneal arter akımlarında zayıflama tespit edilmiş. Aorto-femoral bypass grefti izlenememiştir. Hasta sağ bacak arter anjiyografisi ve endovasküler girişim için kliniğimize yönlendirilmiş. Sol radiyal arter yoldan 6F, $90 \mathrm{~cm}$ açılı kılıf gerektirmeyen kılavuz kateter (ASahi Intecc. S/L) kullanılarak yapılan sağ ayak anjiyografide; sağ external iliak arter çıkışından itibaren tam tıkanıklığın başladığı ve bu tıkanıklığın popliteal artere kadar ilerlediği tespit edildi (Resim- 1a,b). Heparin (5000 IU) bolusundan sonra mikro kateter (Merit Impress Hyd. 4F HH1) ve 0.018"' k1lavuz telle (Asahi Intecc Treasure 12) antegrad penetrasyon denendi fakat başarılı olunamadı. Zira săg external iliak arterin total tıkanıklı̆̆ı, sağ ana iliak arter çıkışından başlamakta ve "blunt" özelliğindeydi. Bunun üzerine, 4F-7cm Prelude sheath (Merit Medica-USA) kullanılarak dorsalis pedis arter kanüle edildi ve retrograd girişime geçildi. 300-cm, 0.018-inch Treasure12 guide wire (Asahi Intecc.Japan) eşliğinde bir 4F, 100-cm düz uçlu kateter (Impress catheter Merit Medica, USA), ön tibial arter üzerinden popliteal artere ilerletildi (Resim-1c). 


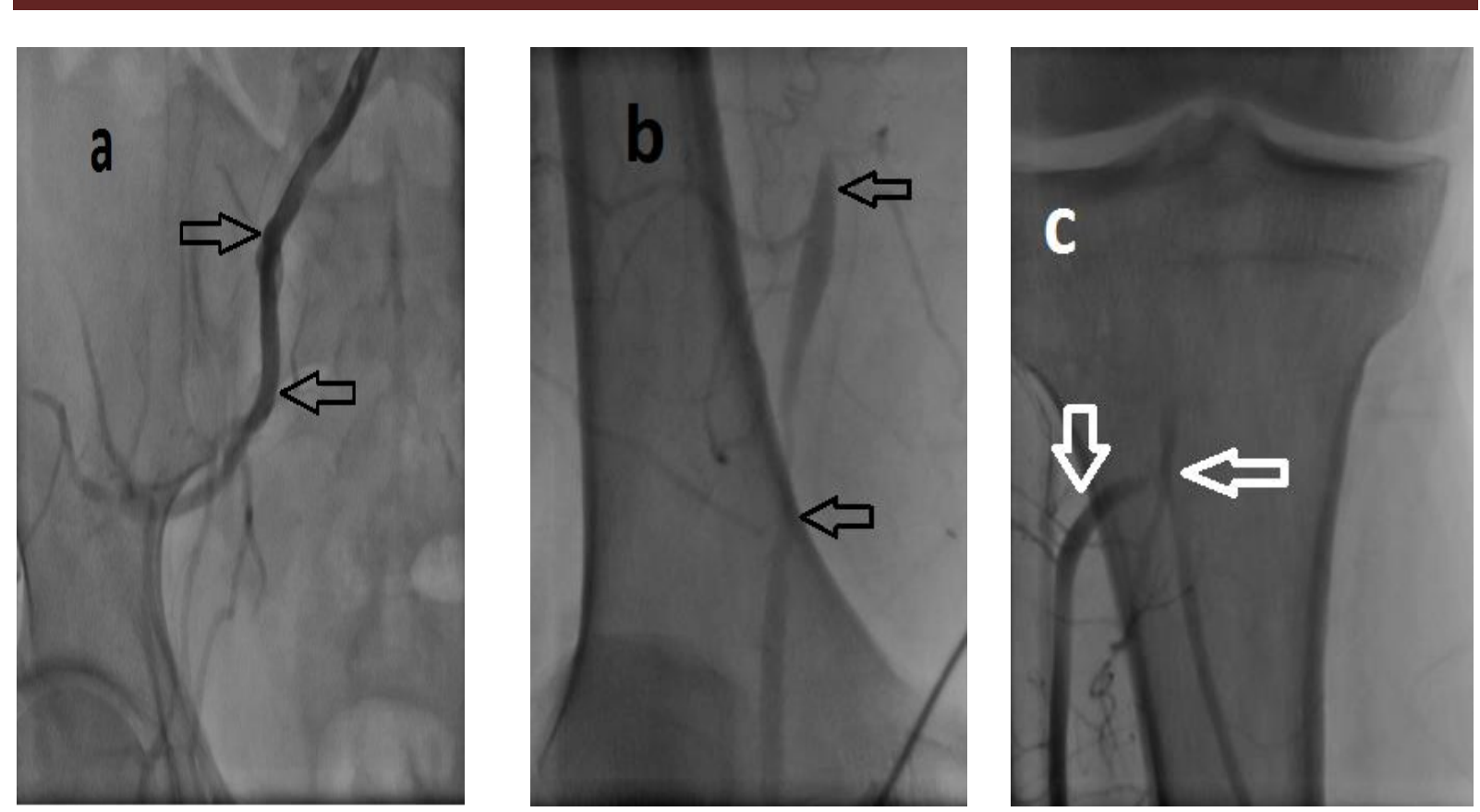

Resim 1: .Sağ ana iliak ater görüntülenmesi: sağ external ilak arter çıkış kısmından tam tıkalı ve internal iliak arter izleniyor (a). Femoral arter uç kısmında zayıf bir doluş ve popliteal arterin başında kritik darlık izleniyor (b). Dorsalis pedis arterden retrograd girişle ön tibial artere kateter yerleştirilip arka tibial ve peroneal arterin görüntülenmesi (c )

$\mathrm{Bu}$ uygulama ile subintimal ilerleme sağlandı. Ancak sağ external iliak arterde lümene düşülemedi. Bunun üzerine 0.014" kalınlığındaki Astato (Asahi Intecc,Japan) periferik kılavuz teline çift açı verilerek lümene ulaşıldı. Mikro kateter ilerletilerek lümen içerisine pozisyonlandırılıp, opak madde verilerek lümen teyidi yapıldı. İçerisinden $300 \mathrm{~cm}$ uzunluğunda 0.018" kalınlığında "floppy" uçlu kılavuz tel (Asahi Intecc- Treasure Floppy, Japan) gönderilerek antegrad kateter içerisine ulaşım sağlandı (Resim-2a). İşlemin antegrat yol üzerinden yapılması için gerekli hazırlıklar yapılırken; radiyal arter üzerinden gönderilecek balon ve stentlerin kateter şaft uzunluklarının femoral ve popliteal düzeyine yetişemeyeceği anlaşıldığından; bu işlemlerin de retrograd hat üzerinden yapılmasına karar verildi. Retrograd yoldan gönderilen 5 x $60 \mathrm{~mm}$ anjiyoplasti balonu (Trireme GilederFlex, USA) ile predilatasyonlar yapılarak rekanalizasyon sağland1. Anjiyografik olarak tatmin edici bir sonuç alınamadığ 1 için stentlemeye karar verildi. Stent implantasyonu için $4 \mathrm{~F}$ kılıf $6 \mathrm{~F}$ (Merit Medica,USA/ Prelude-radyal) ile değiştirildi. Değişik uzunluklarda ve çaplarda 4 adet kendiliğinden açılan nitinol stent (Polaris-Qualimed, Germany) implante edildi. $6.0 \mathrm{~mm}$ balon (Trireme-GliderFlex, USA) ve $7.0 \mathrm{~mm}$ balon (Qualimed-PyxisVQ, Germany) ile post dilatasyonlar yapılarak işlem başarıyla sonlandırıldı (Resim-2b). Kontrol anjiyografide sağ external iliak arterden popliteal artere kadar akım yeniden sağlandı ve diz altı arterlerde güçlü bir akım gerçekleştirildi (Resim-2c). İşlem başarıyla sonlandırıldı. Hasta yatışının 3. gününde 
aspirin, atorvastatin ve klopidogrel tedavisi eşliğinde şifa ile taburcu edildi. Hastaya yaşam tarzı değişikliği ve özellikle sigarayı bırakması önerildi. Hastanın 1. ve 3. ay kontrollerinde şikayetleri geçmişti. Klinik değerlendirmede sağ ayak nabızları dolgun ve devamlı olarak izlendi.
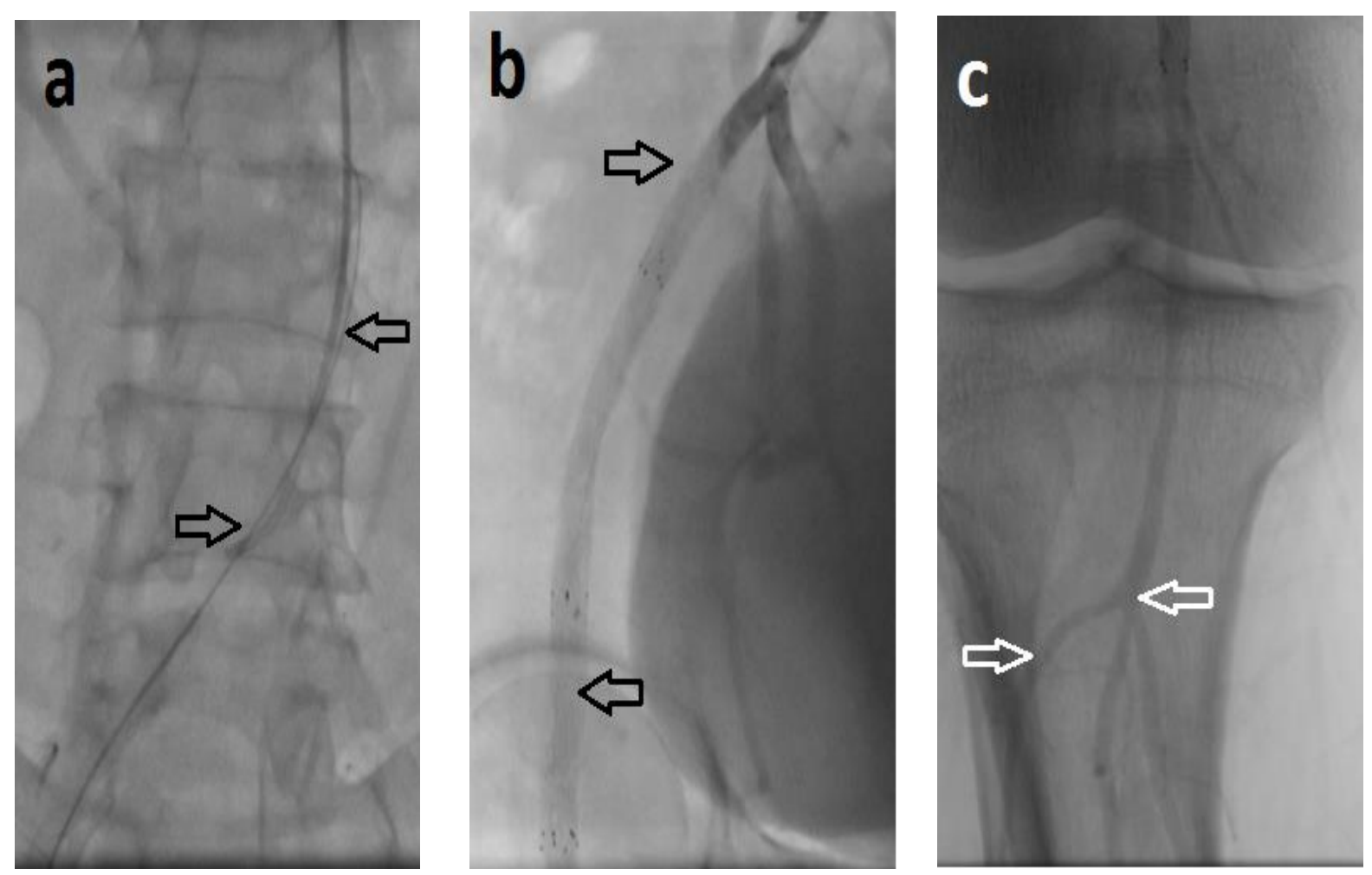

Resim 2: $300 \mathrm{~cm}$ uzunluğunda 0.018 ' kalınlığında "floppy" uçlu kılavuz telin (Asahi Intecc- Treasure Floppy,Japan) retrograd gönderilerek antegrad kateter içerisine yerleştirilmesi (a). Sağ external ve ana femoral arterin stentlenmesi (b). Başarılı rekanalizasyon sonrası diz ve diz altı arterlerde güçlü doluşun izlenmesi (c)

\section{TARTIŞMA}

Sadece geleneksel tedavi uygulanan kritik ayak iskemisi olan hastalarda 1 y1llik amputasyon riski yaklaşık \%70-95 oranındadır. Oysa revaskülarizasyon bu amputasyon riskini \%24-28'e kadar indirebilir (6). Köprüleme cerrahisinin mortalite-morbitite açısından \%4 ile \%37 arasında perioperatif riski vardır (6-7). Perkütan revaskülarizasyon, köprüleme cerrahisine göre daha düşük perioperatif riskler içerir (8). Düşük morbidite (ölüm oranı) ve yüksek teknik başarı oranı (\%90'dan fazla) ile aortoiliak lezyonlar için endovasküler revaskülarizasyon önerilmektedir (9). İliak arter darlığı ve tıkanıklıklarında stentleme önerilmektedir (9). Olgunun özğünlüğü ve uygulayıcının tecrübesi kendiliğinden genişleyebilir stent veya balon seçimini belirleyen faktörlerdir. İliak arter darlıklarında geçici stentlemeye göre düşük 
diseksiyon riski nedeniyle kendiliğinden genişleyebilir stentler tercih edilmektedir (9). $\mathrm{Bu}$ vakada da balon anjiyoplastiyle predilatasyonlar yaptıktan sonra kendiliğinden genişleyebilir stent kullanıldı. Endovasküler girişim esnasında antegrad yaklaşımların yaklaşık \%20'si başarısızlıkla sonuçlanır (10). Femoro popliteal uzun tam tıkanıklıklarda antegrad ve retrograd yaklaşım ile subintimal rekanalizasyon çok başarılı sonuçlar verebilir. Aynı zamanda standart metodların başarısız olduğu durumlarda transkolleteral (11), transpedal $(12,13)$ yollar yada confluent two-balloon tekniği (14) alternatif olarak kullanılabilir. Kasık altı arter tıkanıklıklarında transpedal yaklaşımın uygulandığı vaka sayısı çok azdır $(5,11,12)$. Bu olgu, kasık üstü bir arter tıkanıklığında da transpedal yaklaşımın uygulanabileceğini gösteren çok daha nadir bir vakadır. Bu vakada medial ve lateral plantar arterlere göre daha kolay giriş ve rekanalizasyon imkanı olduğu için dorsalis pedis arteri seçildi. Dorsalis pedis arter ayak sırtında daha düz bir seyir izler. USG eşliğinde daha kolay tespit ve giriş yapılabilir. İster subintimal ister intraluminal olsun retrograd rekanalizasyon başlangıç giriş yeri için nonkalsifik bir segment seçilirse buradan tıkalı artere kullanılabilir bir rekanalizasyon oluşturmak daha kolay olabilir. İşlemin yaklaşı \%2 majör ve \%7,8 oranında minör komplikasyon riski vardır (15). Bu komplikasyonlar arasında; giriş yerinde hematom ve tıkanıkl1k sayılabili. Bu olguda herhangi bir komplikasyon izlenmedi.

Sonuç olarak ayağı tehdit eden kritik arteryel hastalıklarda ayak kurtarıcı girişim kaçınılmazdır. Endovasküler revaskülarizasyon tekniklerinin ve bu alanda kullanılan malzemelerin gelişmesine bağlı olarak perkütan revaskülarizasyon seçeneğinin gelecekte giderek artan sıklıkta kullanıllacağı tahmin edilmektedir. Bu vaka iliak arterlerdeki tıkanıklıklarda da balon-stent işleminin, dorsalis pedis arter kullanılarak retrograd yaklaşımla başarılı bir şekilde uygulanabileceğini gösteren çok nadir bir olgudur. 


\section{KAYNAKLAR}

1. Becker GJ, Katzen BT, Dake MD. Noncoronary angioplasty. Radiology 1989; 170:403-412.

2. Ingl H, Nasim A, Bolia A, et al. Subintimal angioplasty of isolated infrageniculate vessels in lower limb ischemia: long-term results. J Endovasc Ther 2002; 9:411-416.

3. Biancari F, Kantonen I, Alback A, et al. Popliteal-to-distal bypass grafts for critical leg ischemia. J Cardiovasc Surg 2000; 41:281-286.

4. Tonnesen KH, Sager P, Karle A ,Henriksen L, Jorgensen B. Percutaneous transluminal angioplasty of the superficial femoral artery by retrograde catheterization via the popliteal artery. Cardiovasc Intervent Radiol $1988 ; 11: 127-131$.

5. Spinosa DJ, Harthun NL, Bissonette EA, et al. Subintimal arterial flossing with antegrade-retrograde intervention (SAFARI) for subinitimal recanalization to treat chronic critical limb ischemia. J Vasc Intervent Radiol 2005; 16:37-44.

6. Wolfe JH, Wyatt MG. Critical and subcritical ischaemia. Eur J Vasc Endovasc Surg 1997; 13:578-582.

7. Goshima KR, Mills JL, Sr, Hughes JD. A new look at outcomes after infrainguinal bypass surgery: traditional reporting standards systematically underestimate the expenditure of effort required to attain limb salvage. J Vasc Surg 2004; 39:330-335.

8. Faglia E, Dalla Paola L, Clerici G, et al. Peripheral angioplasty as the first-choice revascularization procedure in diabetic patients with critical limb ischemia: prospective study of 993 consecutive patients hospitalized and followed between 1993 and 2003. Eur J Vasc Endovasc Surg 2005; 29:620-627.

9. The Task Force on the Diagnosis and Treatment of Peripheral Artery Diseases of the European Society of Cardiology (ESC). European Heart Journal (2011) 32, 2851-2906 doi:10.1093/eurheartj/ehr211

10. Sidhu R, Pigott J, Pigott M, et al. Subintimal angioplasty for advanced lower extremity ischemia due to TASC II C and D lesions of the superficial femoral artery. Vasc Endovascular Surg 2010; 44:633-637.

11. Fusaro M, Agostoni P, Biondi-Zoccai G. "Trans-collateral" angioplasty for a challenging chronic total occlusion of the tibial vessels: a novel approach to percutaneous revascularization in critical lower limb ischemia. Catheter Cardiovasc Interv 2008; 71:268-272.

12. Manzi M, Fusaro M, Ceccacci T, et al. Clinical results of below-the knee intervention using pedal-plantar loop technique for the revascularization of foot arteries. J Cardiovasc Surg (Torino) 2009; 50:331-337.

13. Fusaro M, Dalla Paola L, Biondi-Zoccai G. Pedal-plantar loop technique for a challenging below-the-knee chronic total occlusion: a novel approach to percutaneous revascularization in critical lower limb ischemia. J Invasive Cardiol 2007;19:E34-E37.

14. Ikushima I, Hirai T, Ishii A, et al. Confluent two-balloon technique: an alternative method for subintimal recanalization of peripheral arterial occlusion. J Vasc Interv Radiol 2011; 22:1139-1143.

15. Miguel Montero-Baker et al. Retrograde approach for complex popliteal and tibioperoneal occlusions. J Endovasc Ther 2008; 15:594-604. 